\title{
ERRATUM
}

INDUSTRIAL HEALTH

(2007) Vol. 45, No. 4, Pages: 512-519.

\section{Design of Digital Filters for Frequency Weightings Required for Risk Assessments of Workers Exposed to Vibration}

Andrew N. RIMELL and Neil J. MANSFIELD

Department of Human Sciences, Loughborough University, Loughborough, Leicestershire, LE11 3TU, UK

Due to a typographical error, Equation 4 was presented incorrectly. It should have been:

$H_{s}(s)=\frac{s^{2}+\frac{\omega_{5}}{Q_{5}} s+\omega_{5}^{2}}{s^{2}+\frac{\omega_{6}}{Q_{6}} s+\omega_{6}^{2}}$

All subsequent equations are correct. 